\title{
Characterisation of the MutS and MutL Proteins from the Pseudomonas avellanae Mismatch Repair (MMR) System
}

\author{
${ }^{I}$ Dipartimento di Biologia, Università di Roma Tor Vergata, Italy \\ ${ }^{2}$ Unità di Ricerca per la Frutticoltura, C.R.A. Caserta, Italy \\ ${ }^{3}$ Centro di Ricerca per la Frutticoltura, C.R.A. Roma, Italy \\ ${ }^{4}$ Istituto di Biologia e Patologia Molecolari del CNR, Roma, Italy
}

Lucia Grenga $^{1}$, Fabio Gervasi ${ }^{2}$, Luciano Paolozzi ${ }^{1}$, Marco Scortichini ${ }^{3}$ and Patrizia Ghelardini ${ }^{4, *}$

\begin{abstract}
The identification and analysis of the Pseudomonas avellanae mismatch repair system (MMR) were performed via sequencing and cloning the $m u t S$ and $m u t L$ genes and then analyzing the characteristics of the corresponding proteins studying their function and biological role in an E. coli heterologous system. In these studies, the $P$. avellanae MutS and MutL proteins were shown to localise at the nucleoid level, in a MutS-dependent manner as far as MutL is concerned, and were also able to complement the defect observed in both the mutS and mutL knockout strains of $E$. coli. In addition, their ability to form both homo and heterodimers between each other was shown by using the prokaryotic two-hybrid assay. Our results represent a first step to elucidate the MMR mechanism in plant pathogenic pseudomonads since the MMR genes were identified in $P$. syringae pathovars but there was no evidence on their action as effective repair products.
\end{abstract}

Keywords: Cytologic localisation, MMR system, MutL protein, MutS protein, Pseudomonas avellanae, two-hybrid assay.

\section{INTRODUCTION}

Pseudomonas avellanae is the causative agent of bacterial canker and responsible for the decline of Corylus avellana L. This bacterium severely damages cultivated hazelnuts in northern Greece [1] and central Italy [2]. The main symptom observed in the fields is a sudden wilting of twigs, branches, and the tree. The disease is particularly prevalent during the late spring and summer.

It was reported [3] that $P$. avellanae, isolated directly from diseased hazelnut specimens in central Italy, showed, in some cases, a colony phase variation with the presence of transparent colony variants (i.e., water drop-like colonies) on nutrient agar medium supplemented with $5-7 \%$ sucrose (NSA). These variants appeared encapsulated, were possibly not pathogenic and did not induce a hypersensitivity reaction in tobacco leaves. Reversal towards typical $P$. avellanae colonies (i.e., creamy-white colour) was also observed following the re-streaking of transparent variants onto NSA plates [3].

Van den Broek et al., [4, 5] reported that MutS-dependent mismatch repair (MMR) is involved in Pseudomonas sp. PCL 1171 colony phase variation. The process occurs via the accumulation of spontaneous reversible mutations in the regulatory genes $g a c A$ and $g a c S$, which belong

*Address correspondence to this author at the Istituto di Biologia e Patologia Molecolari del CNR, Roma, Italy, c/o Dipartimento di Biologia, Università di Roma Tor Vergata, Via della Ricerca Scientifica, 1, I00133 Roma, Italy; Tel: (+39) 06 72594243; E-mail: ghelardini@bio.uniroma2.it to the two-component system that affects the production of secondary metabolites [6]. Indeed, mutations in the mutS gene increased the frequency of switching from phase I (opaque colonies) to phase II (translucent colonies) up to 1000 -fold for the high frequency of mutations accumulated in $g a c A / S$. These results indicate that the level of MutSdependent repair is a determinant for the frequency of phase variation.

On the basis of these results and to investigate the characteristics of phase variation in $P$. avellanae, we preliminarily undertook an analysis of its mismatch repair system (MMR) by identifying and analysing its $m u t S$ and mutL genes.

Unlike the MutHLS pathway of Enterobacteriaceae [7], Oliver et al., [8] showed that the MMR system in $P$. aeruginosa lacks the mutH gene. Only mutS and $m u t L$ genes are present, and, as expected, their alteration is associated with a "mutator" phenotype. Although Pseudomonas aeruginosa does not have the corresponding E. coli MutH and Dam homologues, and consequently the MMR system seems to work differently, Jacquelín et al., [9] showed that the $m u t L$ gene from $P$. aeruginosa is able to complement a MutL-deficient strain of $E$. coli.

No prior studies were performed to examine the MMR system of plant pathogenic pseudomonads and all the available information (i.e., $m u t S$ and $m u t L$ gene sequences) stemmed from the complete genome sequencing of representative species, including several of the $P$. syringae pathovars. Additionally, as expected, no gene homologus of $m u t H$ has been identified. 


\section{MATERIALS AND METHODS}

\section{Bacterial Media and Chemicals}

Nutrient broth (NB) and nutrient agar (Difco) supplemented with $5 \%$ sucrose (NSA) were used for bacterial culture and plating of $P$. avellanae. LB broth [10] was used for culturing and plating E. coli. SM (salt solution) was used for bacterial dilutions, as described by Miller [10]. The antibiotics used (Sigma) were ampicillin $\left(50 \mu \mathrm{g} \mathrm{ml}^{-1}\right)$, tetracycline $\left(40 \mu \mathrm{g} \mathrm{ml}^{-1}\right)$, kanamycin $\left(30 \mu \mathrm{g} \mathrm{ml}^{-1}\right)$, and nalidixic acid $\left(40 \mu \mathrm{g} \mathrm{ml}^{-1}\right)$. Synthetic oligonucleotides (Sigma) used for $E$. coli and $P$. avellanae gene amplifications and those used for $P$. avellanae mut $S$ and mutL gene sequencing are listed in Table $\mathbf{1}$.

\section{Bacterial Strains and Plasmids}

Pseudomonas avellanae and E. coli K-12 strains as well as all plasmids used in this study are listed in Table $\mathbf{2}$.

\section{DNA Sequencing and Sequence Analysis}

The putative $P$. avellanae mutS and mutL genes were obtained by PCR amplification performed with synthetic oligonucleotides whose sequence derived on the analysis of the $P$. syringae pathovars phaseolicola, syringae and tomato sequence alignments. Because the 5' and 3' sequences of both genes from these pathovars are strongly conserved, we used the first and the last 20 nucleotides of the $P$. syringae mutS and mutL genes to design the oligonucleotides. The PCR products were then cloned in pGemT easy vector (Promega) to obtain pGemT-mut $S_{\text {P.av }}$ and pGemT-mut $L_{\text {P.av }}$ respectively.

The mutS and mutL gene sequences from Pseudomonas avellanae strain CRA-ISFN1 were obtained by sequencing the pGemT-mut $S_{\text {P.av }}$ and pGemT-mut $L_{\text {P.av }}$ vectors with primers 1-7 and 1, 2, 8 and 9 (Table 1), respectively. All sequences were determined at BMR Genomics.

\section{Plasmid Construction and Bacterial Two Phages Two- hybrid Assay (TP-THA)}

Recombinant plasmids (listed in Table 2) for the TPTHA were constructed by cloning the genes of interest in $\mathrm{pcI}_{\mathrm{P} 22}$ and $\mathrm{pcI}_{434}$ vectors. The DNA of the $P$. avellanae mut genes, obtained by PCR amplification using specific oligonucleotides carrying the SalI and BamHI restriction sites at the ends, listed in Table $\mathbf{1}$, was cloned in the same sites of the $\mathrm{pcI}_{\mathrm{P} 22}$ and $\mathrm{pcI}_{434}$ vectors, respectively [11]. All of the different combinations of recombinant plasmids coding for the chimerical repressors, obtained as described above, were transformed into the recipient $E$. coli strain R721, which carries the 434/P22 chimerical operator that governs the lac $Z$ expression as reporter gene. $\beta$-galactosidase activity was assayed as described by Miller [10]. Bacterial cultures were grown at $37^{\circ} \mathrm{C}$ in LB medium supplemented with 0.1 $\mathrm{mM}$ isopropyl- $\beta$-D-thiogalactopyranoside (IPTG) to an $\mathrm{OD}_{600}$ of 0.3 . $\beta$-galactosidase activity was evaluated for each strain and normalised to the value of $E$. coli R721 without plasmids. Residual $\beta$-galactosidase activity less than $50 \%$ was indicative of a protein-protein interaction, whereas activity greater than $50 \%$ indicated an uncertain or null interaction. The rationale for this cut-off is reported in previous studies [11-13]. E. coli $\mathrm{R} 721$ with the $\mathrm{pcI}_{434} 434$ plasmid was used as a negative control and $E$. coli R721 with both the $\mathrm{pcI}_{\mathrm{P} 22} 434$ and $\mathrm{pcI}_{434} 434$ plasmids (Table 2) was used as a positive control.

\section{Construction of GFP Derivatives for Microscopic Observation}

The $P$. avellanae and E. coli MutS and MutL proteins fused with Aequorea victoria green fluorescent protein

Table 1. Synthetic Oligonucleotides used in this Study

\begin{tabular}{|c|c|c|}
\hline 1 & $\mathrm{p}_{22}-31$ SalI $\mathrm{F}$ & 5'-CATTGATTAGCATGGTTAGAGC-3' \\
\hline 3 & mutS P.avellanae $800 \mathrm{R}$ & 5'-TCCAGGTTGCGACGGCTGGC-3' \\
\hline 4 & mutS P.avellanae $801 \mathrm{~F}$ & 5'-GCTGGACACCAACCTGTCCGG-3' \\
\hline 6 & mutS P.avellanae $1600 \mathrm{R}$ & 5'-TCAGGTCTTCGAGCAACGTCT-3' \\
\hline 7 & mutS P.avellanae $1601 \mathrm{~F}$ & 5'-TCGACCACCTTGCGCCGCTGC-3' \\
\hline 8 & mutL P.avellanae $850 \mathrm{R}$ & 5'-CGATACGCCTGACGCACCGCGT-3' \\
\hline 12 & mutL P.syringae $1 \mathrm{~F}$ & 5'-ATGACCGATCTTCTCCTCGA-3' \\
\hline 13 & mutL P.syringae $1938 \mathrm{R}$ & 5'-TCATTGACCGCGCAGGAATA-3' \\
\hline
\end{tabular}


Table 2. Bacterial Strains and Plasmids used in this Study

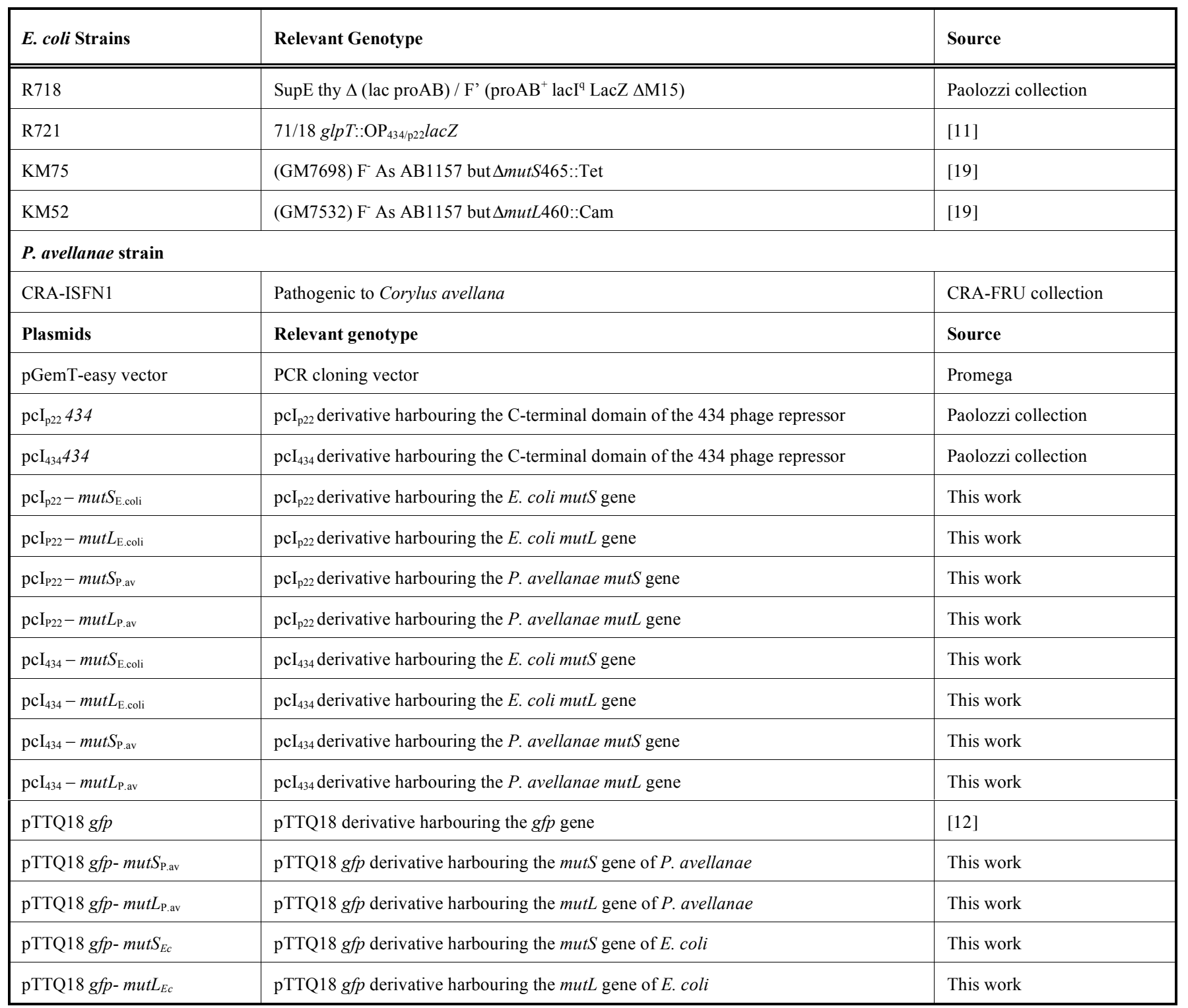

(GFP) were obtained by in-frame cloning of the PCRproduced DNA fragments of the corresponding genes. These were constructed in the Pst I and HindIII sites, downstream of the GFP gene previously inserted in the EcoRI and Pst $\mathrm{I}$ sites of plasmid pTTQ18, to give the plasmid pTTQ18-GFP [12].

\section{Fluorescent Microscopy}

Cultures of E. coli KM75 $\Delta m u t S$ strain, harbouring the recombinant plasmids containing the genes of interest fused in-frame with GFP, were grown in LB supplemented with the appropriate antibiotics up to $\mathrm{OD}_{600}$ values in the range of 0.3 to 0.4 . The addition of $0.1 \mathrm{mM}$ IPTG induced the expression of the tagged genes, and the culture was further grown for $1 \mathrm{~h}$ at $30^{\circ} \mathrm{C}$. Next, $1 \mu \mathrm{L}$ of 4', 6-diamino-2phenylindole (DAPI, $5 \mathrm{mg} \mathrm{mL}^{-1}$ ) was added to a $100 \mu \mathrm{L}$ aliquot of bacterial culture for $10 \mathrm{~min}$ in the dark. The sample was then centrifuged and resuspended in the same volume of saline buffer. Finally, $5 \mu \mathrm{L}$ were loaded on poly-
L-lysine-coated slides and examined using a Delta vision (AppliedPrecision) Olympus 1 X 70 microscope.

The $P$. avellanae and $E$. coli MutL localisations were examined in the E. coli KM75 strain in the absence and presence of $P$. avellanae MutS, coded by the $\mathrm{pcI}_{434} m u t S_{\text {P.av }}$ plasmid.

\section{Complementation Experiments}

Cultures of E. coli KM75 $\Delta m u t S$ strain, transformed with $\operatorname{pcI}_{\mathrm{P} 22} m u t S_{\text {P.av }}$ and $\mathrm{pcI}_{\mathrm{P} 22} m u t S_{\mathrm{E} . \mathrm{coli}}$, respectively, were grown in LB supplemented with the appropriate antibiotics up to an $\mathrm{OD}_{600}$ equal to 0.9 and then plated on LB containing 40 $\mu \mathrm{g} / \mathrm{ml}$ of nalidixic acid. The frequency of nal-resistant clones was compared to both the mut deleted strains without plasmids and to the R718 wild-type strain.

The same kind of experiments was performed with $E$. coli KM52 $\Delta m u t L$ transformed with $\operatorname{pcI}_{\mathrm{P} 22} m u t L_{\mathrm{P} . \mathrm{av}}$ and $\operatorname{pcI}_{\mathrm{P} 22} m u t L_{\mathrm{E} . \mathrm{coli}}$, respectively. 


\section{RESULTS AND DISCUSSION}

To investigate whether the characteristics of phase variation in $P$. avellanae reported by Scortichini and Angelucci [3] could be related to an alteration of the MutS-dependent mismatch repair (MMR) system [4, 5], we preliminarily undertook a study of its mismatch repair system (MMR) by analysing the mutS and mutL genes.

Although MutH, MutL and MutS are essential components of the mismatch repair system in Escherichia coli only mutS and $m u t L$ genes are found in most organisms, whereas the $m u t H$ gene is limited to some proteobacteria [14] and, in particular, Pseudomonas species lack the $m u t H$ homologue [15]. In fact, no homologous sequence was found for the endonuclease MutH, necessary for cleaving the DNA strand with the mismatch in the E. coli MMR system, thus indicating that a different endonuclease is probably performing the cleavage in $P$. aeruginosa [8].

\section{Identification of the Putative $P$. avellanae mutS and mutL Genes}

Since the complete genome sequence is not available for $P$. avellanae, the synthetic oligonucleotide sequences used to amplify the putative $P$. avellanae mutS and mutL genes were obtained by the analysis of sequence alignments from $P$. syringae pathovars phaseolicola, syringae and tomato.
Because the 5' and 3' sequences of both genes from these pathovars are strongly conserved, we used the first and the last 20 nucleotides of the $P$. syringae mutS and mut $L$ genes to design the oligonucleotides. The DNA obtained by PCR amplification from a culture of $P$. avellanae strain CRAISFN1 with the two pairs of oligonucleotides, 10-11 and 1213 , for $m u t S$ and $m u t L$, respectively, (Table 1) was cloned into the pGemT-easy vector and sequenced.

The $P$. avellanae strain CRA-ISFN1 mutS and mutL genes were entirely sequenced. The sequences were deposited at NCBI under the accession numbers AM982525 for $m u t S$ and AM982526 for $m u t L$. The two gene sequences were roughly similar in size to the corresponding genes of $P$. syringae pathovar syringae (i.e., $2601 \mathrm{bp}$ compared to 2568 for mutS and $1944 \mathrm{bp}$ compared to 1938 for mutL, respectively). The phylogenetic analysis is enclosed as supplementary data file.

Screening of the putative MutS amino acid sequence revealed that the main differences between the $P$. avellanae and the $P$. syringae pathovars are in positions 367 to 373 , where, in $P$. avellanae, an insertion of seven amino acids (EPGARPR) is present. The putative MutL protein shows a $96 \%$ identity to the $P$. syringae pathovars, with the main difference of a 2-residues (QY) insertion in positions 414-415.

A)
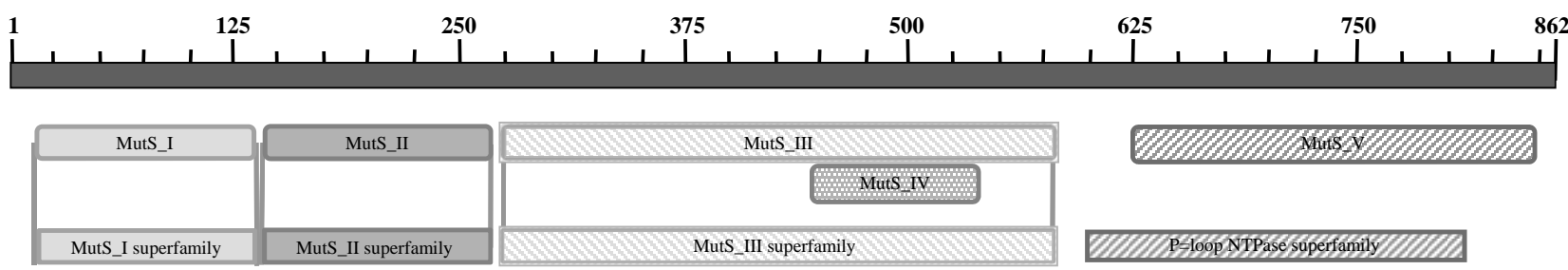

MutS_I superfamily

MutS_III superfamily

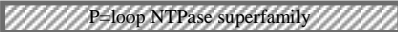

B)

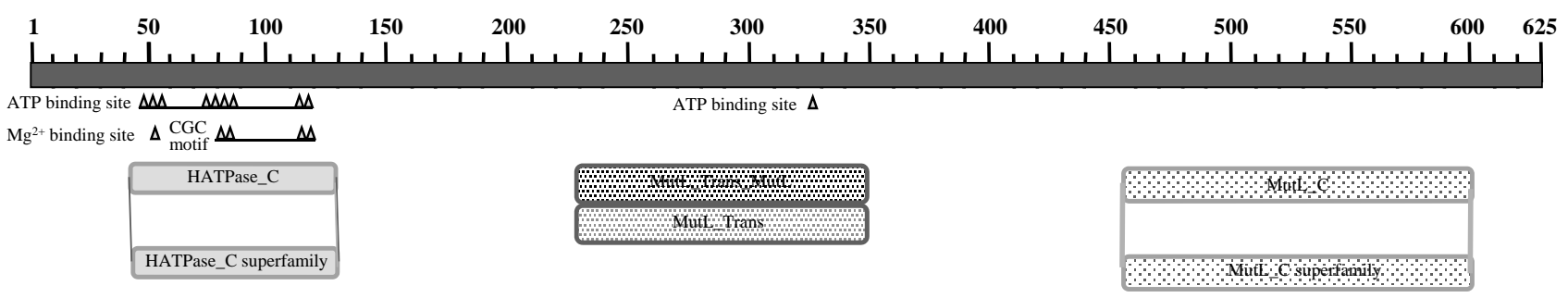

Fig. (1). Structural domains of $P$. avellanae mismatch proteins. A) MutS: The aligned regions of the protein correspond: (i) with globular domain I (residues 12 to 124), which is involved in DNA binding. In this domain, there are two copies of the typical mismatch-binding motif, F-X-E, at positions 37 to 39 and 41 to 43 of the protein, as in P. syringae. In both cases, the sequence is FYE, as in E. coli, where only one copy is localised at position 36 to 38. (ii) with domain II (residues 132 to 257) defined as connector domain; (iii) with domain III or core domain (residues 263 to 572); (iv) in part with globular domain IV or clamp domain, which is also involved in DNA binding (residues 438 to 528); and (v) with domain V, which contains a Walker A motif and is structurally similar to ABC transporter ATPase domains (residues 588 to 801). B) MutL: For MutL, residues 43 to 129 constitute an ATP binding domain that contains two G-X-G motifs, present in the conserved domain of HATPase-C and located in loops defining the top and bottom of the ATP binding pocket. The transducer MutL_Trans_MutL domain extends from residue 228 to residue 349. Also, the C-terminal region of the protein (residues 456 to 601 ) defines the MutL C domain, which is involved in the homodimerisation of the protein. 
A search for conserved $P$. avellanae putative MutS and MutL protein domains using RPS-BLAST with clusters of orthologous groups of proteins $[16,17]$ revealed typical Mut family domains in both cases (Fig. 1).

\section{Study of the P. avellanae MMR System}

Since it was impossible to address directly to the question whether the two putative genes identified constitute the $P$. avellanae MMR system by generating and analyzing the behaviours of the mutants, due to the lack of suitable genetic tools in this organism, to assess their role in the MMR system, we studied their behaviour in an E. coli heterologous system.

The MMR mechanism predicts that, during initiation of DNA mismatch repair, a monomer of the mismatch recognition protein, MutS, after binding the mismatch on the DNA, homodimerises and then recruits MutL in the presence of ATP. The MutS (ATP)-MutL ternary complex forms and then further activates the repair process [reviewed in 18]. A consequence of this model is that MutL is unable to bind the DNA and therefore does not have the ability to localise at the nucleoid level in the absence of MutS.

To reconstruct the MMR system of $P$. avellanae, we studied the ability of MutS and MutL to interact individually and in combination, and to localise at the nucleoid level in the bacterial cell. The complementation of E. coli mutS- and mutL-deleted strains and the study of the $P$. avellanae protein interactions with those of $E$. coli (where the MMR system is well characterised) were performed to assess their effective functionality as DNA repair proteins.

\section{i) Interactions of the P. avellanae MutS and MutL Proteins between Each Other and with Those of E. coli}

The ability of $P$. avellanae MutS and MutL to form homodimers and to interact between each other was studied using the prokaryotic two phages two-hybrid assay (TPTHA) [11]. In the TP-THA system, the interaction between two proteins is tested by their ability to form a chimeric lambdoid repressor. When the gene products interact they form a functional repressor that shuts down the expression of a chromosomal copy of the lac $Z$ reporter gene, whose synthesis is governed by a hybrid 434-P22 promoter/operator region [11]. $\beta$-galactosidase synthesis, which is constitutive in the strain without plasmids, is repressed in the presence of the two plasmids only if the two proteins under investigation interact to form a functional chimeric repressor. The $\beta$ - galactosidase activity of the E. coli strain R721 harbouring the pairs of plasmids was evaluated and normalised to that of the parental strain without plasmids. Residual activity less than $50 \%$ is indicative of an interaction between the two proteins $[12,13]$.

The two P.avellanae mut genes were cloned in frame with the N-terminal part of the lambdoid phage repressors 434 and $\mathrm{P} 22$ in the $\mathrm{pcI}_{434}$ and $\mathrm{pcI}_{\mathrm{P} 22}$ plasmids to form the recombinant plasmids, $\mathrm{pcI}_{434}-m u t S$, $\mathrm{pcI}_{\mathrm{P} 22}-m u t S, \mathrm{pcI}_{434}-m u t L$, and $\operatorname{pcI}_{\mathrm{P} 22}-m u t L$. In addition, to investigate the possible interactions between $P$. avellanae and their orthologous $E$. coli mismatch repair proteins, the E. coli mut genes were cloned in the same vectors described above. The assays were performed as described in Materials and Methods. The results represent the average of at least three independent determinations.

The $\beta$-galactosidase activity of the bacterial strain without plasmids was 2300 Miller units, and if it harbours only one of the two plasmids the residual activity was in the order of $100 \%$.

The results (Table 3) suggest that, as expected from the behaviour of the Mut proteins, both the $P$. avellanae MutS and MutL proteins are able to homodimerise and also to interact with each other. In addition, although phylogenetically different, the $P$. avellanae MMR proteins were shown to be interchangeable with their homologous $E$. coli proteins during the interaction analyses.

Table 4. Complementation of E. coli mut Deleted Strains

\begin{tabular}{|l|l|}
\hline Strain & Frequency of nal ${ }^{\mathrm{R}}$ Cells \\
\hline \hline $\mathrm{R} 718$ & $2.0 \times 10^{-6}$ \\
\hline $\mathrm{KM} 75 \Delta m u t S$ & $1.0 \times 10^{-3}$ \\
\hline $\mathrm{KM} 75 / \mathrm{pcI}_{\mathrm{P} 22-m u t S_{\mathrm{Ec}}}$ & $1,2 \times 10^{-6}$ \\
\hline $\mathrm{KM} 75 / \mathrm{pcI}_{\mathrm{P} 22-m u t S_{\mathrm{P} . \mathrm{av}}}$ & $3,2 \times 10^{-5}$ \\
\hline $\mathrm{KM} 52 \Delta$ mutL & $1.0 \times 10^{-3}$ \\
\hline $\mathrm{KM} 75 / \mathrm{pcI}_{\mathrm{P} 22-m u t L_{\mathrm{Ec}}}$ & $1.4 \times 10^{-6}$ \\
\hline $\mathrm{KM} 75 / \mathrm{pcI}_{\mathrm{P} 22-m u t L_{\mathrm{P} . a v}}$ & $5.0 \times 10^{-5}$ \\
\hline
\end{tabular}

The results represent the mean of three independent experiments.

Table 3. Interaction of Pseudomonas avellanae MutS and MutL Proteins between Each Other and with the Orthologous Escherichia coli Proteins

\begin{tabular}{|l|l|l|l|l|}
\hline \multicolumn{1}{|c|}{ pcI $_{\text {434 }}$} & MutS $_{\text {P.av }}$ & MutL $_{P . a v}$ & MutS $_{\text {E.coli }}$ & MutL $_{\text {E.coli }}$ \\
\hline \hline MutS $_{P . a v}$ & $38 \% \pm 3.4$ & & & \\
\hline MutL $_{P . a v}$ & $17 \% \pm 2.3$ & $24 \% \pm 4.3$ & & \\
\hline MutS $_{E . \text { coli }}$ & $36 \% \pm 4.0$ & $16 \% \pm 1.9$ & $34 \% \pm 3.3$ & $28 \% \pm 2.0$ \\
\hline MutL $_{E . \text { coli }}$ & $28 \% \pm 1.8$ & $41 \% \pm 2.5$ & $11 \% \pm 4.1$ & \\
\hline
\end{tabular}




\section{ii) Complementation of E. coli mutS and mutL Deletion Mutants by P. avellanae Mut Proteins}

Despite the phylogenetic distance between the E. coli and $P$. avellanae MMR proteins (see the supplementary data), the MutS proteins alignment shows about $57 \%$ identity that is mostly clustered on domains I and IV of the protein
(Fig. 1). The identity decreases to $41 \%$ when the two MutL proteins are compared. In this case the best alignment is observed on the first part of the protein.

The sequence homology between the $P$. avellanae and $E$. coli Mut proteins and the results observed in the TP-THA suggest that the $P$. avellanae mismatch proteins could be

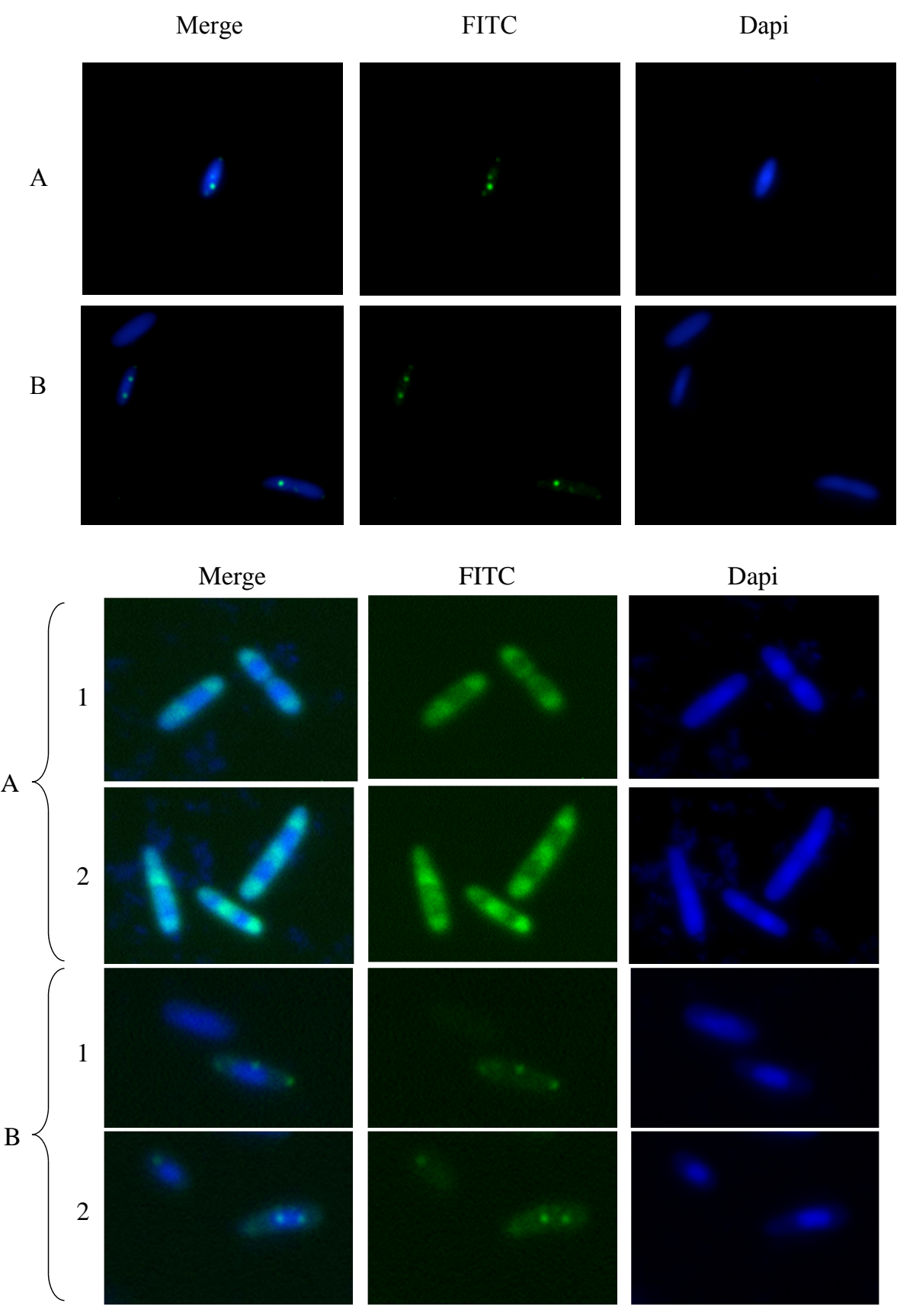

Fig. (2). Mut proteins localization at the nucleoid level. A) Fluorescence micrographs illustrating the localisation of $P$. avellanae and E. coli MutS in the E. coli $\Delta m u t S$ strain. The experiments were performed as described in Materials and Methods. Cells were stained with DAPI to visualise DNA (blue). The GFP (green) highlights MutS and the merged images show the localisation of $P$. avellanae (A) and E. coli MutS (B) on the E. coli chromosome. B) Fluorescence micrographs illustrating the localisation of $P$. avellanae and $E$. coli MutL in the $E$. coli $\Delta m u t S$ strain. The experiments were performed as described in Materials and Methods. Cells were stained with DAPI to visualise DNA (blue). The GFP (green) highlights MutL and the merged images show the localisation of MutL on the E. coli chromosome in the absence (A) or in the presence (B) of P. avellanae MutS.

1) Localization of $P$. avellanae MutL.

2) Localization of E. coli MutL. 
functional in $E$. coli. For these reasons, we studied their ability to complement the defects of E. coli mutS and mutL deletion mutants. The E. coli strains deleted for the mutS and mutL genes show an increased mutation rate due to the loss of DNA repair. This phenotype can be evaluated by studying the mutation rate frequency of a particular marker, such as an antibiotic resistance derived by a mutation of the antibiotic target gene on the bacterial chromosome. In our experiments, we studied the frequency of nalidixic acid resistance in both the mutated and wild type strains, in the presence and absence of recombinant plasmids coding for the $P$. avellanae and $E$. coli mutS and mutL genes. The results of these studies are shown in Table 4 . The frequency of nalidixic acid resistance increases about 1,000 fold in the $\Delta m u t s S$ and $\Delta m u t L$ strains when compared to the wild-type strain. The defects of the mut-deleted strains are fully rescued in the presence of recombinant plasmids carrying the E. coli mut genes. Additionally, the presence of the $P$. avellanae proteins can rescue, almost completely, the wild-type phenotype, confirming the results obtained using the twohybrid assay.

These results indicate that the gene products identified as putative Mut proteins in $P$. avellanae are effectively involved in the MMR repair process and suggests that the $P$. avellanae Mut proteins are interchangeable with that of $E$. coli in performing the mismatch repair functions.

\section{iii) Localisation of P. avellanae MutS and MutL in E. coli.}

MutS localisation on the bacterial chromosome at the mismatch level is the first step in the repair process and is followed by the recruitment of MutL [18]. The onset of the $P$. avellanae MMR system was characterised studying the ability of its MutS protein to localise in the E. coli KM75 $\Delta m u t S$ strain. This design was chosen to avoid any interference with the endogenous MutS protein.

As shown by Fig. (2A), P. avellanae MutS is able to localise in the $E$. coli at the same extent as the $E$. coli protein, whereas, according to the MMR model of action, in the absence of MutS either the $P$. avellanae or the E. coli, MutL does not appear to be condensed in foci, but diffused in the cytoplasm. On the other hand, the presence of $P$. avellanae MutS on a plasmid allows for both $P$. avellanae and $E$. coli MutL migration towards the nucleoid (Fig. 2B).

The ability of $P$. avellanae MutS to recruit the $E$. coli MutL protein on the chromosome is consistent with the result of the observed interactions between the $E$. coli and $P$. avellanae MMR proteins.

\section{CONCLUSION}

The characterisation of the $P$. avellanae Mut system is an early step in the study of its possible involvement in the colony phase variation of bacteria isolated from Corylus avellana plants infected by $P$. avellanae [3].

In their whole, our results confirmed that the gene sequences, caught by PCR amplification with the synthetic oligonucleotides designed on the $P$. syringae, correspond to MMR genes. Moreover, the coded proteins are interchangeable with the MutS-L proteins of $E$. coli. In addition, they represent a first step to elucidate the MMR mechanism in plant pathogenic pseudomonads since, until now, the MMR genes were identified in $P$. syringae pathovars but no evidence on their action as effective repair products was produced.

\section{CONFLICT OF INTEREST}

Declared none.

\section{ACKNOWLEDGEMENTS}

This work was partially financed by NeCS society and "Progetti e piani per le Frontiere Tecnologiche nell'ambito dei progetti di Ricerca, Sviluppo e Innovazione delle PMI" supported by FILAS Lazio (Italy).

\section{SUPPLEMENTARY MATERIAL}

Supplementary material is available on the publishers Web site along with the published article.

\section{REFERENCES}

[1] Psallidas PG, Panagopoulos CG. A bacterial canker of hazelnut in Greece. Phytopatholologische Zeitschrift 1979; 94: 103-11.

[2] Scortichini M. Bacterial canker and decline of hazelnut. Plant Disease 2002; 86: 704-9.

[3] Scortichini M, Angelucci L. Phenotypic characterization of Pseudomonas avellanae (Psallidas) Janse et al. and occurrence of colony variants. J Plant Pathol 1999; 81: 55-61.

[4] van den Broek, D, Chin-A-Woeng TFC, Bloemberg GV, Lugtenberg BJJ. Role of RpoS and MutS in phase variation of Pseudomonas sp. PCL1171. Microbiology 2005; 151: 1403-8.

[5] van den Broek D, Chin-A-Woeng TF, Bloemberg GV, Lugtenberg BJ. Molecular nature of spontaneous modifications in gacS which cause colony phase variation in Pseudomonas sp. strain PCL1171. J Bacteriol 2005; 187: 593-600.

[6] van den Broek D, Chin-A-Woeng TFC, Eijkemans K, Mulders IH, Bloemberg GV, Lugtenberg BJJ. Biocontrol traits of Pseudomonas spp. are regulated by phase variation. Mol Plant Microbe Interact 2003; 16: 1003-12.

[7] Hsieh P. Molecular mechanisms of DNA mismatch repair. Mutat Res 2001; 486: 71-87.

[8] Oliver A, Sánchez JM, Blázquez J. Characterization of the GO system of Pseudomonas aeruginosa. FEMS Microbiol Lett 2002; 217: 31-5.

[9] Jacquelín DK, Filiberti A, Argaraña CE, Barra JL. Pseudomonas aeruginosa MutL protein functions in Escherichia coli. Biochem J 2005; 388: 879-87.

[10] Miller JH. Experiments in Molecular Genetics. Cold Spring Harbor, NY: Cold Spring Harbor Laboratory 1972.

[11] Di Lallo G, Fagioli M, Barionovi D, Ghelardini P, Paolozzi L. Use of a two-hybrid assay to study the assembly of a complex multicomponent protein machinery: bacterial septosome differentiation. Microbiology 2003; 149: 3353-9.

[12] D'Ulisse V, Fagioli M, Ghelardini P, Paolozzi L. Three functional subdomains of the Escherichia coli FtsQ protein are involved in its interaction with the other division proteins. Microbiology 2007; 153: 124-38.

[13] Fadda D, Santona A, D'Ulisse V, et al. Streptococcus pneumoniae DivIVA: localization and interactions in a MinCD-free context. J Bacteriol 2007; 189: 1288-98.

[14] Friedhoff P, Sheybani B, Thomas E, Merz C, Pingoud A. Haemophilus influenzae and Vibrio cholerae genes for mutH are able to fully complement a mutH defect in Escherichia coli. FEMS Microbiol Lett 2002; 208: 123-8.

[15] Ramos JL, Ed. Pseudomonas Kluwer Academic/Plenum Publishers: New York, NY 2004; vol. 1-3. 
[16] Tatusov RL, Natale DA, Garkavtsev IV, et al. The COG database: new developments in phylogenetic classification of proteins from complete genomes. Nucleic Acids Res 2001; 29: 22-8.

[17] Tatusov RL, Fedorova ND, Jackson JD, et al. The COG database: an updated version includes eukaryotes. BMC Bioinformatics 2003; 11: 4-41.
[18] Schofield MJ, Hsieh P. DNA mismatch repair: molecular mechanisms and biological function. Annu Rev Microbiol 2003; 57: 579-608.

[19] Murphy KC. Use of bacteriophage lambda recombination functions to promote gene replacement in Escherichia coli. J Bacteriol 1998; 180: 2063-71.

(C) Grenga et al.; Licensee Bentham Open.

This is an open access article licensed under the terms of the Creative Commons Attribution Non-Commercial License (http://creativecommons.org/licenses/ by-nc/3.0/) which permits unrestricted, non-commercial use, distribution and reproduction in any medium, provided the work is properly cited. 